\title{
Limitation of tachycardia zone resulting from longitudinal dissociation of the atrioventricular node in concealed pre-excitation
}

\author{
LASZLO LITTMANN, * ROBERT H SVENSON \\ From the Department of Cardiology and the Heineman Medical Research Center, Charlotte Memorial Hospital and \\ Medical Center, Charlotte, North Carolina, USA
}

SUMmary Two cases with a concealed left-sided accessory atrioventricular bypass tract are described. In both, functional longitudinal dissociation of the atrioventricular node narrowed the range of atrial premature beat coupling intervals which could initiate re-entry using the accessory pathway. In case 1 early premature atrial beats were followed by an atrioventricular nodal re-entrant echo. The atrial echo pre-empted retrograde conduction over the Kent bundle and thus limited the development of paroxysmal supraventricular tachycardia. In case 2 atrioventricular nodal conduction showed typical features ascribed to dual atrioventricular nodal pathways. In addition there was a bradycardia-related retrograde block in the concealed accessory pathway. Early premature atrial beats, because of exclusive "slow pathway" anterograde conduction, arrived at the ventricles during the period of bradycardia-dependent retrograde block and failed to initiate a macro re-entrant tachycardia. This study shows that (1) longitudinal dissociation within the atrioventricular node may limit the ability to initiate tachycardia in patients with concealed pre-excitation; and (2) discontinuous atrioventricular nodal conduction curves occasionally help to reveal bradycardia-related retrograde block in a concealed accessory pathway.

Discontinuous atrioventricular nodal conduction curves and atrioventricular nodal re-entry are common electrophysiological responses to premature atrial stimulation in man. ${ }^{1}$ The former are believed to reflect dual atrioventricular nodal pathways and the latter dual pathways, functional longitudinal dissociation, or reflection within the atrioventricular node. ${ }^{2}$ These phenomena have recently been described in a few patients who also had manifest ${ }^{3-5}$ or concealed pre-excitation. ${ }^{6-9}$ In most of these reported cases the atrioventricular nodal conduction characteristics were unrelated to the initiation or maintenance of the paroxysmal supraventricular tachycardia. ${ }^{6-8}$ In some, the critical atrioventricular conduction delay required for macro re-entry was achieved by block in the fast atrioventricular nodal pathway, and thus initiation of the tachycardia was enhanced by "duality" of atrioventricular conduction. $^{59}$ In a few patients with an accessory pathway, atrioventricular nodal re-entry itself proved to be the mechanism of the tachycardia. ${ }^{4} 810$

The purpose of the following report is (1) to present

* Dr Littmann is a research fellow of the Heineman Medical Research Center. Parent Institution: Semmelweis University Medical School, Budapest, Hungary. Received for publication 10 December 1980 two unusual cases of concealed pre-excitation where atrioventricular nodal re-entry and dual atrioventricular nodal conduction limited rather than enhanced initiation of paroxysmal supraventricular tachycardia with early atrial extrasystoles, and (2) to show for the first time, a bradycardia-related retrograde block in a concealed accessory pathway.

\section{Case reports}

Case 1, a 57-year-old man, and case 2, a 54-year-old woman, were studied because of long histories of paroxysmal supraventricular tachycardia. Electrophysiological evaluation of the patients included intracardiac recordings from several sites and incremental pacing and extrastimulation. ${ }^{6} 911$ The two cases had the following features in common. (1) Routine electrocardiograms were normal. (2) Ventricular pre-excitation was not produced by right or left atrial pacing and extrastimuli. (3) The presence of a left-sided accessory atrioventricular pathway capable of retrograde conduction only could be proved by ventriculoatrial conduction studies and endocardial atrial mapping. ${ }^{611}$ (4) The concealed accessory atrioventricular pathway was responsible 

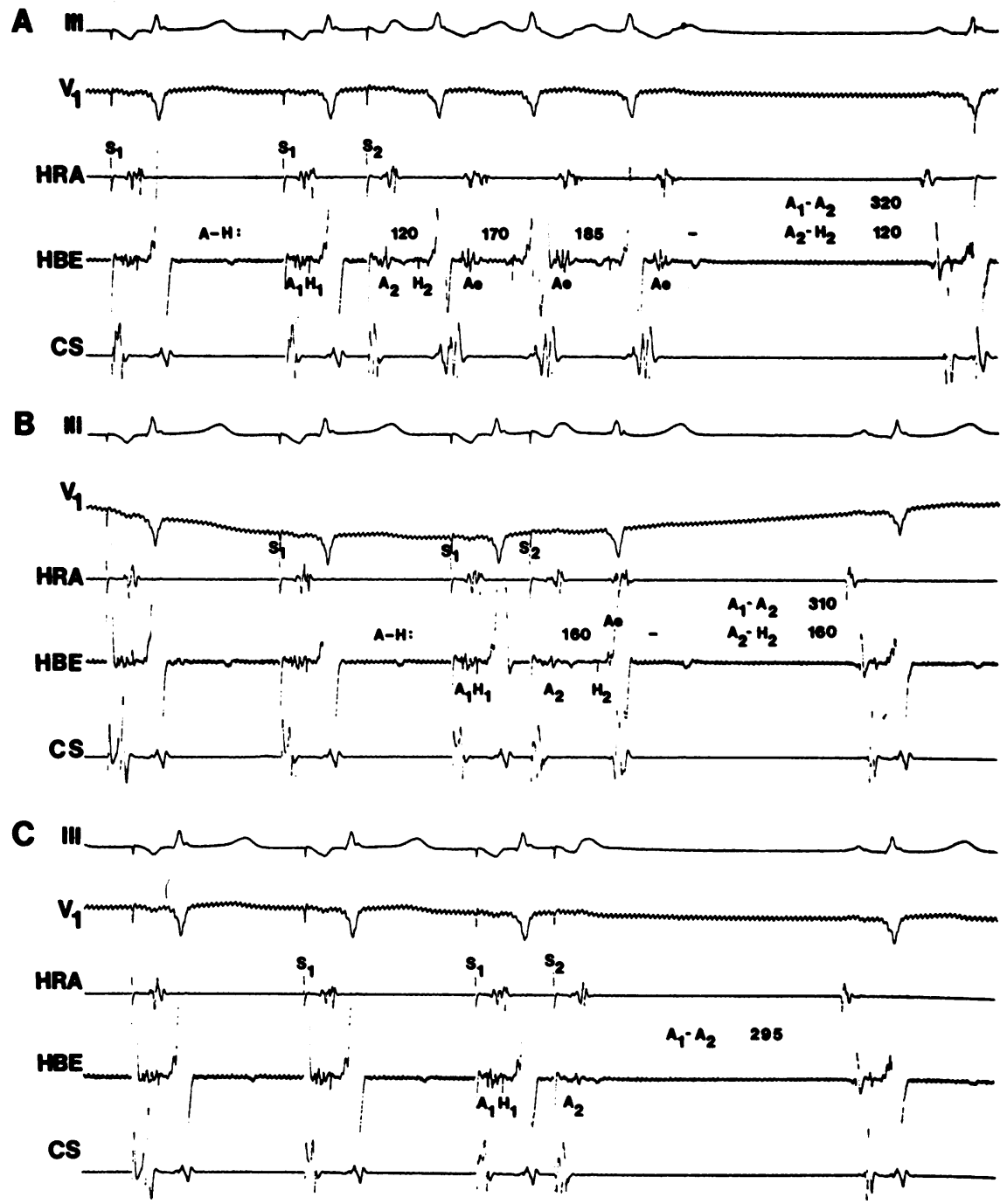

Fig. 1 (case 1) Limitation of the tachycardia zone by atrioventricular nodal re-entry. Left atrial pacing from the coronary sinus is performed at a basic cycle length of $600 \mathrm{~ms}$. In A, a non-sustained supraventricular tachycardia resulting from macro re-entry over a left-sided atrioventricular bypass tract is initiated by a premature atrial impulse with coupling time of $320 \mathrm{~ms}$. Note the left-to-right activation sequence of the atrial echo beats (Ae). In $\mathrm{B}$, the coupling time is $310 \mathrm{~ms}$. After prolongation of the $A_{2}-\mathrm{H}_{2}$ interval to 160 $\mathrm{ms}$, there is a single atrioventricular nodal re-entant complex, and macro re-entry does not occur. Note the simultaneous atrial and ventricular activation and the low-to-high right atrial activation sequence of the echo in $\mathrm{B}$. Panel $\mathrm{C}$ shows that blocked premature stimuli are not followed by atrial echoes.

Each panel is arranged from top to bottom as follows: electrocardiographic lead III, chest lead $I\left(V_{1}\right)$, high right atrial lead $(H R A)$, His bundle lead $(H B E)$, and coronary sinus lead $(C S) . S_{1}, A_{1}$, and $H_{1}$ are, respectively, the stimulus artefact, low right atrial deflection, and His deflection of the last basic drive beat; $S_{2}, A_{2}$, and $\mathrm{H}_{2}$ are the respective deflections of the atrial extra stimulus. Paper speed in each illustration is $100 \mathrm{~mm} / \mathrm{s}$. All numbers in this and subsequent figures are in ms. 
A

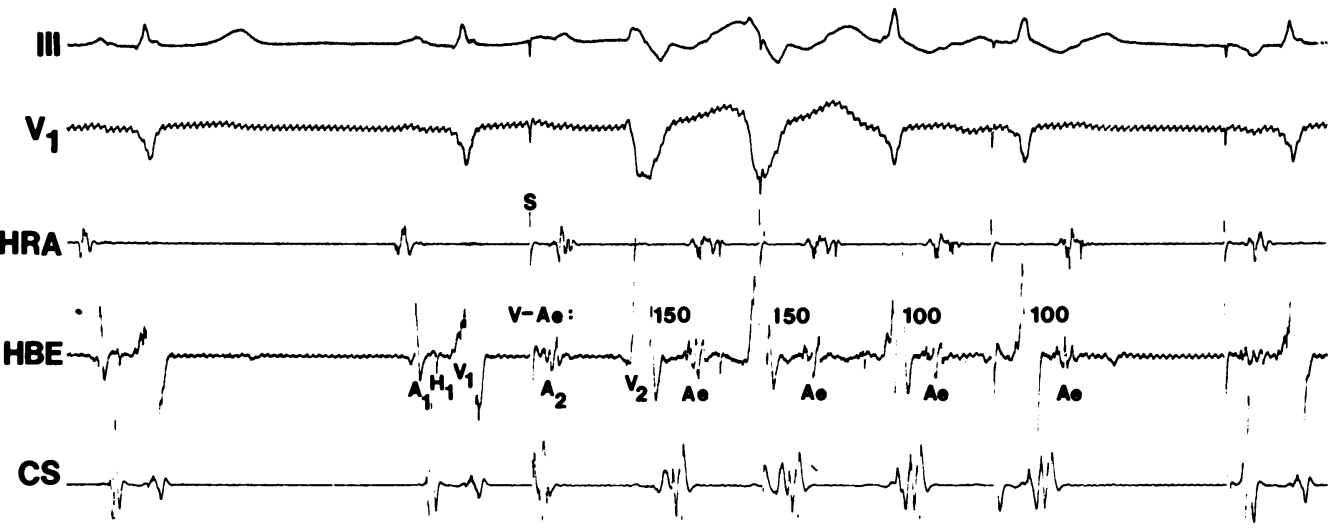

B

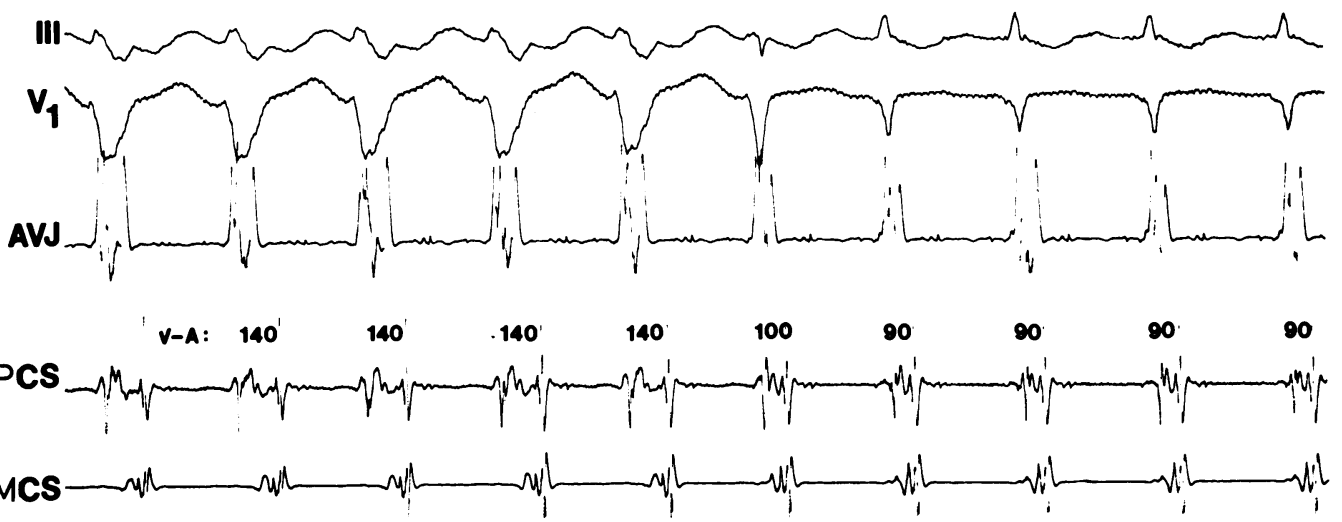

Fig. 2 (case 1) Macro re-entry incorporating a left-sided accessory pathway. In panel A, the tachycardia is initiated after a long ventricular cycle. Ventriculoatrial conduction times in the first two complexes with left bundle-branch block are 50 ms longer than in complexes with normal intraventricular conduction. There are two ineffective stimulus artefacts in this panel. In $\mathrm{B}$, the $V-A$ intervals during the left bundle-branch block tachycardia are $50 \mathrm{~ms}$ longer than during narrow $Q R S$ tachycardia. The cycle length is unchanged because of longer atrioventricular nodal transit times during normal intraventricular conduction.

In panel $\mathrm{A}, C S$ is a mid-coronary sinus lead. In panel $\mathrm{B}$, intracardiac recordings are displayed from top to bottom in the following sequence: bipolar recording from the atrioventricular junction area (AVF); proximal coronary sinus recording $(P C S)$; and mid-coronary sinus recording (MCS).

for the paroxysmal supraventricular tachycardia in both patients. ${ }^{611} 12$ (5) Functional longitudinal dissociation of the atrioventricular node with early atrial extrasystoles limited the potential tachycardia zone with regard to initiation of atrioventricular macro re-entry.

In case 1 , evidence for participation in the paroxysmal supraventricular tachycardia of a leftsided accessory pathway included the following. ${ }^{612}$ (1) The earliest atrial activation during the tachycardia was recorded at the mid-coronary sinus, and this preceded the low right atrial electrogram in the His bundle lead by $40 \mathrm{~ms}$ (Fig. $1 \mathrm{~A}$ and $2 \mathrm{~A}$ ). (2) Ventriculoatrial conduction times during tachycardia were $50 \mathrm{~ms}$ longer on beats with left bundle-branch block than on beats with normal intraventricular conduction (Fig. 2). In case 2, the following data supported macro re-entry over a concealed left-sided accessory tract. $^{6} 12$ (1) The left atrial electrogram recorded at the distal coronary sinus during the tachycardia preceded the low right atrial electrogram by $50 \mathrm{~ms}$ (Fig. 3B and 4). (2) Single extrasystoles delivered at the right ventricular apex during tachycardia pre-excited the left atrium by up to $60 \mathrm{~ms}$. This occurred at a time when the ventricular extrasystole must have found the normal atrioventricular nodeHis bundle pathway refractory from anterograde conduction of the previous beat (Fig. 4).

Analysis of the range of $A_{1}-A_{2}$ intervals producing tachycardia in case 1 disclosed the following (Fig. 1). 
A III

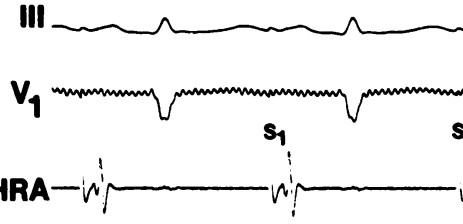

$s_{1} \quad s_{2}$<smiles>[V][V]</smiles><smiles>[C]1C=[Ge]C1</smiles>

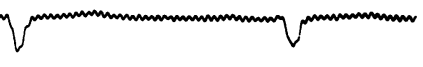

$$
\text { HRA-ri }
$$

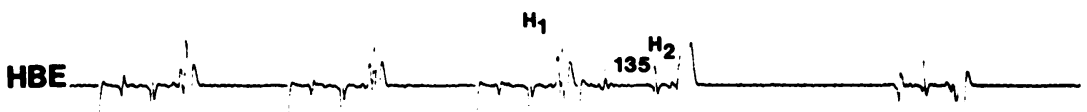

$A_{1} 280 \quad A_{2}$

CS

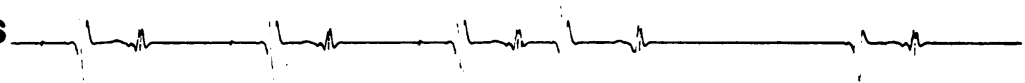

$B$ III r
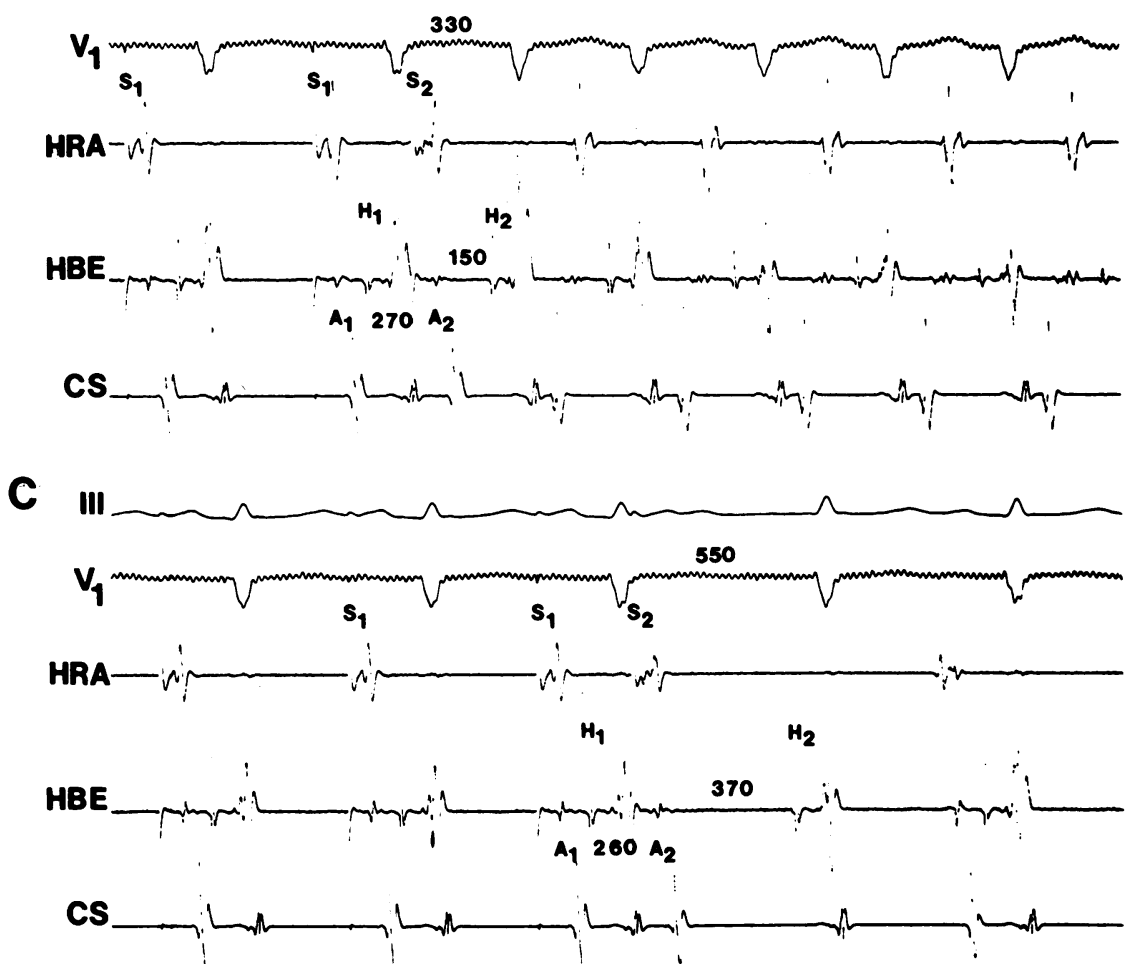

Fig. 3 (case 2) Limitation of the tachycardia zone because of a sudden "jump" in the A-H interval of an early premature beat. Right atrial pacing is performed at a basic cycle length of $500 \mathrm{~ms}$. In B, supraventricular tachycardia using a concealed left-sided accessory pathway is initiated. With a coupling time $10 \mathrm{~ms}$ longer (A), the critical atrioventricular delay for re-entry is not yet achieved. With a coupling time $10 \mathrm{~ms}$ shorter (C), there is a sudden jump in the $\mathrm{A}_{2}-\mathrm{H}_{2}$ interval and again there is no tachycardia. Abbreviations are the same as in Fig. 1 . CS is a distal coronary sinus lead. Numbers above $V_{1}$ leads are $V_{1}-V_{2}$ intervals. 
A

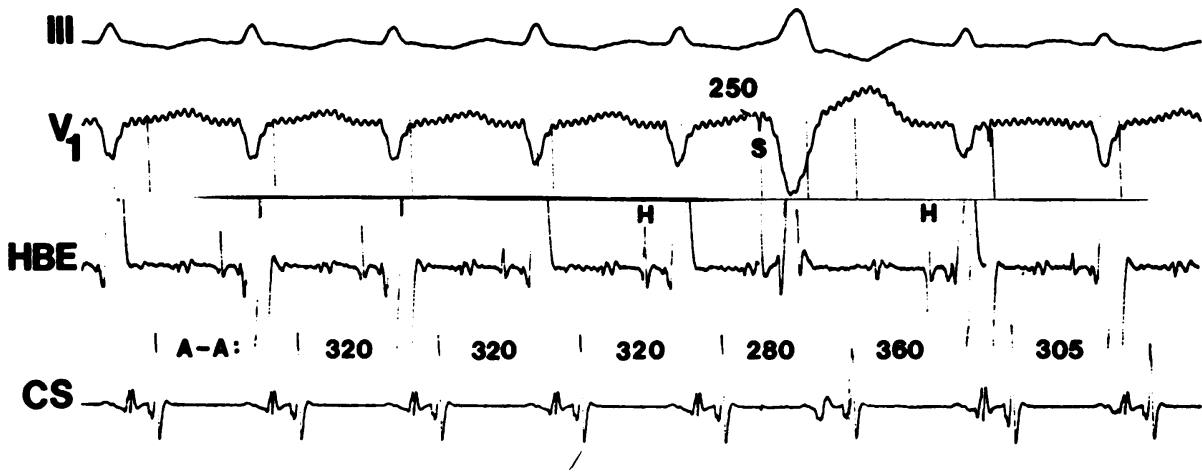

B

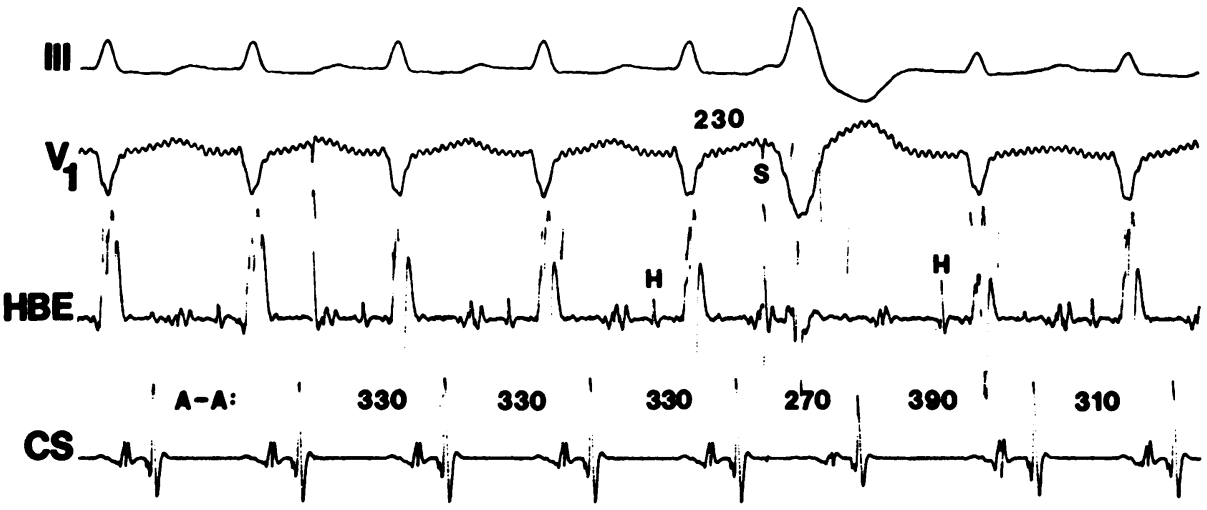

Fig. 4 (case 2) Atrioventricular re-entry using a left-sided atrioventricular bypass tract. A right ventricular extrasystole delivered during the tachycardia at a time when the His bundle was refractory pre-excited the left atrium by $60 \mathrm{~ms}$. Coupling time of the extrasystole was $230 \mathrm{~ms}$. The His deflections bridging the extrasystole are labelled. Note also that the left atrial deflections recorded at the distal coronary sinus precede the low right atrial deflections in the His bundle lead by $50 \mathrm{~ms}$.

When left atrial pacing was performed at a cycle length of $600 \mathrm{~ms}$, the paroxysmal supraventricular tachycardia could be induced by atrial extrasystoles over a narrow range of $A_{1}-A_{2}$ intervals (330-315 ms). The atrioventricular nodal conduction times on these premature beats measured 115 to $130 \mathrm{~ms}$. When $A_{1}-A_{2}$ was decreased to $310-300 \mathrm{~ms}$, the $A_{2}-H_{2}$ interval further increased (Fig. 1B). Nevertheless, these early extrastimuli failed to initiate the tachycardia. The inability of these extrasystoles to induce macro re-entry was secondary to appearance of an atrial echo which pre-empted retrograde conduction over the accessory pathway. The mechanism of the atrial echo was atrioventricular nodal re-entry rather than intra-atrial re-entry since (a) the atrial echo beat had a low-to-high right atrial activation sequence (Fig. 1B); (b) a critical AH interval of $160 \mathrm{~ms}$ was required for the echo to appear (Fig. 1B); and (c) the atrial echo never occurred when $\mathrm{A}_{2}$ blocked in the atrioventricular node (Fig. 1C). Significant intra-atrial delay of the premature impulses could not be held responsible for retrograde block in the accessory pathway. Therefore, it is reasonable to assume that had functional atrioventricular nodal dissociation and atrioventricular nodal re-entry not occurred, the tachycardia zone, at least at the cycle length tested, would have ranged from $330-300 \mathrm{~ms}$. Atrioventricular nodal re-entry was responsible for narrowing of the tachycardia zone by $15 \mathrm{~ms}$.

In case 2, a paroxysmal supraventricular tachycardia incorporating the concealed accessory pathway was initiated over a narrow range of $A_{1}-A_{2}$ intervals. With right atrial pacing at a cycle length of $500 \mathrm{~ms}$, the tachycardia zone ranged from $275-260 \mathrm{~ms}$ (Fig. 3 and 5). By decreasing $A_{1}-A_{2}$ to $260-230 \mathrm{~ms}$, there was a sudden increase in the $A_{2}-H_{2}$ intervals (Fig. 3 and 5). This was probably the result of block in a "fast" and exclusive conduction through a "slow" atrioventricular nodal pathway. ${ }^{1}$ Whenever there was very slow conduction in the atrioventricular node, the 


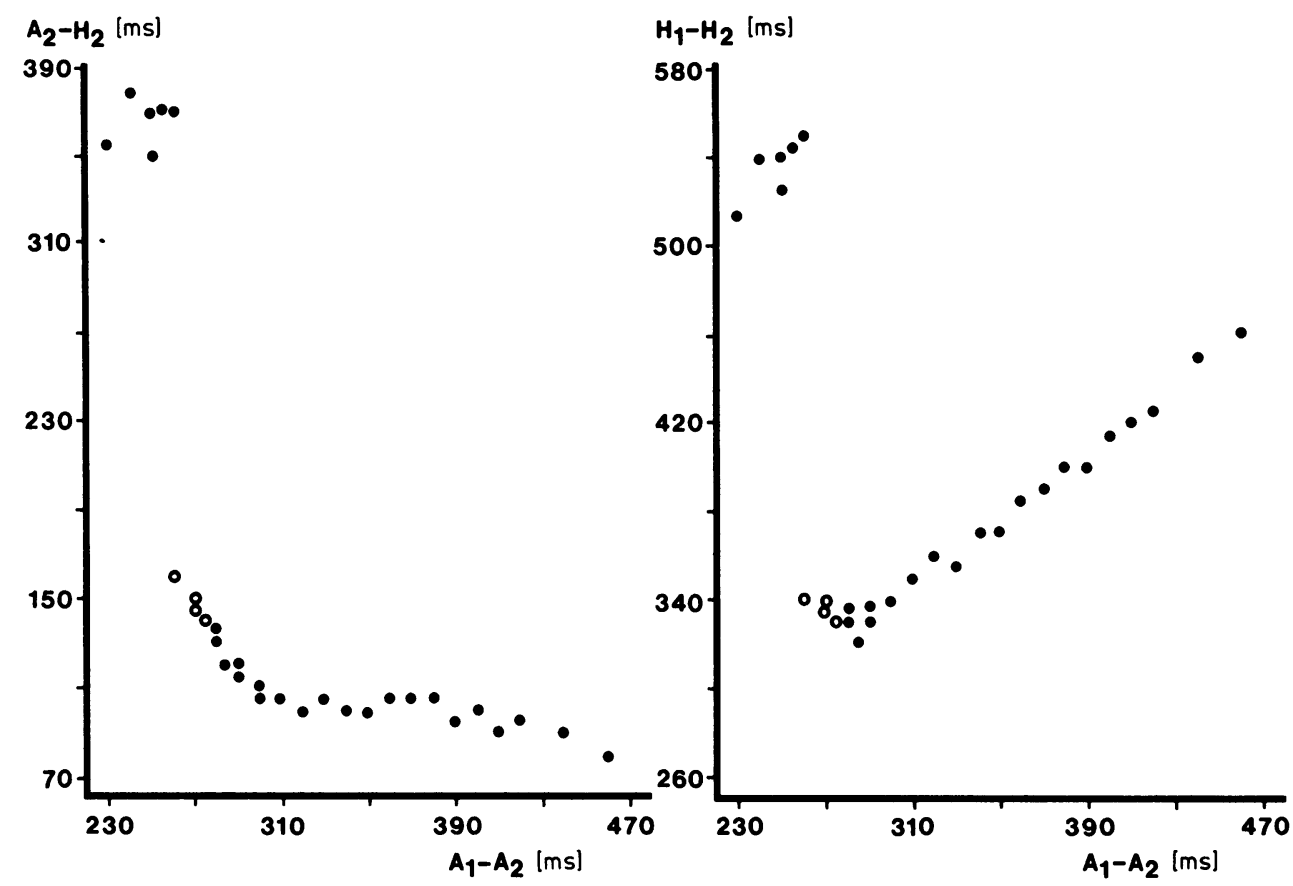

Fig. 5 (case 2) Discontinuous atrioventricular nodal conduction curves suggest "dual atrioventricular nodal pathways". The basic atrial cycle length is $500 \mathrm{~ms}$. Open circles indicate premature atrial stimuli followed by echoes over a concealed accessory bundle. The echo zone is only 15 ms wide.

extrastimulus failed to initiate the tachycardia (Fig. 3C). Atrioventricular nodal re-entrant complexes were also not observed.

The only mechanism that could reasonably explain the above findings is to postulate a bradycardia-dependent retrograde block in the accessory pathway. If this were the case, early premature beats, by conducting exclusively through a slow atrioventricular nodal pathway, would reach the ventricles at a period when the concealed accessory atrioventricular pathway was unresponsive because of the bradycardia-related block. This hypothesis was tested by the ventricular extrastimulus technique. The pertinent findings are illustrated in Fig. 6. Left atrial pacing was performed at the same cycle length as during the anterograde studies. After eight basic drive beats a right ventricular extrasystole was delivered. This methodology was employed to allow the atria to recover fully after the last basic drive stimulus. Thus, retrograde conduction of the ventricular extrasystole was solely dependent on the ability of the normal and anomalous pathways to deliver the impulses to the atria. Ventricular extrasystoles late in diastole all conducted to the atria. The $V_{2}-A_{2}$ intervals progressively increased with increasing prematurity. The low right atrial electrogram $\left(A_{2}\right)$ preceded the left atrial electrogram ( $\mathrm{LA}_{2}$ ) by a constant interval, $40 \mathrm{~ms}$. This suggested that retrograde conduction of late ventricular premature systoles occurred through the atrioventricular node. ${ }^{11} 13$ The shortest coupling time of $V_{2}$ showing this retrograde activation sequence was $430 \mathrm{~ms}$ (Fig. 6A). When $V_{1}-V_{2}$ was decreased to 420 ms, retrograde conduction failed to occur (Fig. 6B). The first supraventricular complex following $V_{2}$ in this panel is a sinus node escape. There was complete retrograde block between coupling times of 420-350 ms (Fig. 6B and 6C). With a $\mathrm{V}_{1}-\mathrm{V}_{2}$ of $340 \mathrm{~ms}$ retrograde conduction reappeared, but this time the atrial activation sequence was left to right (Fig. 6D). This suggested that retrograde conduction occurred over the accessory pathway. ${ }^{11} 13$ This type of conduction was manifest down to a $V_{1}-V_{2}$ interval of $310 \mathrm{~ms}$ (Fig. 6E). With a $\mathrm{V}_{1}-\mathrm{V}_{2}$ of $300 \mathrm{~ms}$ or less, there was again complete retrograde block (Fig. 6F).

The above findings can be interpreted as evidence for bradycardia-dependent retrograde block in the concealed accessory pathway. With $V_{1}-V_{2}$ intervals of $350 \mathrm{~ms}$ or longer, probably all premature ventricular beats blocked in the accessory pathway. The constant $A_{2}-L_{2}$ intervals down to a $V_{1}-V_{2}$ of $430 \mathrm{~ms}$ and complete ventriculoatrial block between $V_{1}-V_{2}$ 's of $420-350 \mathrm{~ms}$ favour this assumption. ${ }^{11}{ }^{13}$ At coupling 
intervals shorter than $350 \mathrm{~ms}$, retrograde conduction occurred exclusively over the accessory pathway until its refractory period of $300 \mathrm{~ms}$ was reached. These findings show a remarkable correlation with the anterograde studies. Whenever a paroxysmal supraventricular tachycardia was initiated by a premature atrial impulse, the $\mathrm{V}_{1}-\mathrm{V}_{2}$ interval was in the range where retrograde conduction could occur through the bypass tract (Fig. 3 and 5). Whenever there was exclusive "slow pathway" anterograde conduction, the $V_{1}-V_{2}$ intervals fell in the range of bradycardia-dependent retrograde block. Atrioventricular re-entry was not initiated (Fig. 3C and 5). Fig. 6G illustrates that bradycardia-dependent retrograde

A

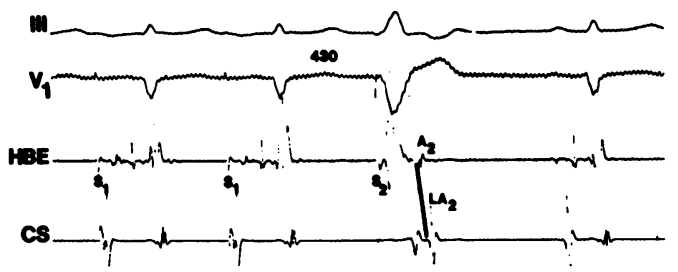

B
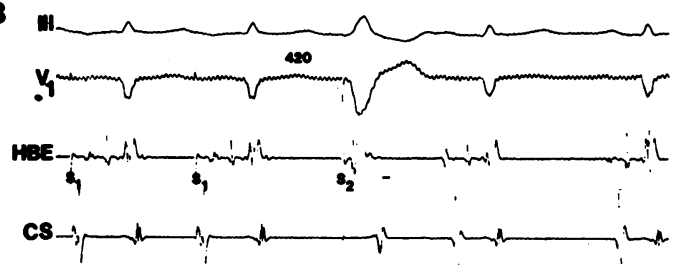

C
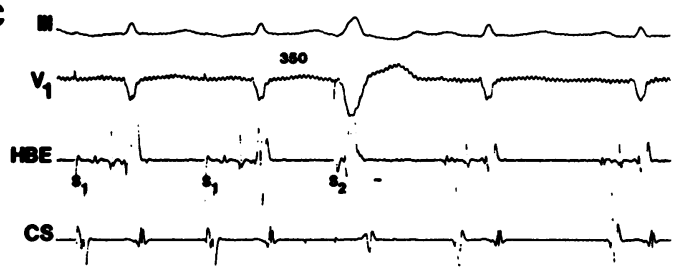

D

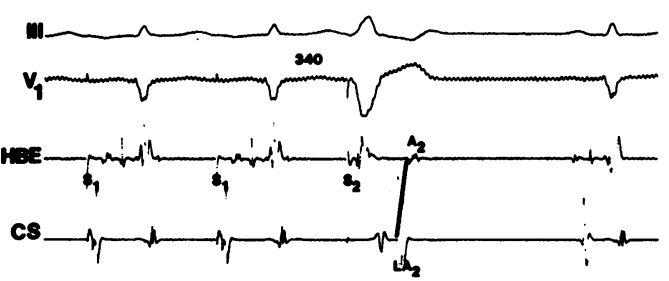

block was a function of the basic cycle length. During tachycardia an atrial extrastimulus which caused a subsequent $\mathrm{V}-\mathrm{V}$ interval of $590 \mathrm{~ms}$ did not result in retrograde block in contrast to long $\mathrm{V}-\mathrm{V}$ intervals during regular pacing at a longer cycle length than the tachycardia. The exact ranges of retrograde accessory pathway conduction at the rate of the tachycardia were not tested by the ventricular extastimulus method.

\section{Discussion}

In the presence of a concealed anomalous bypass tract macro re-entry can be induced by premature atrial

E

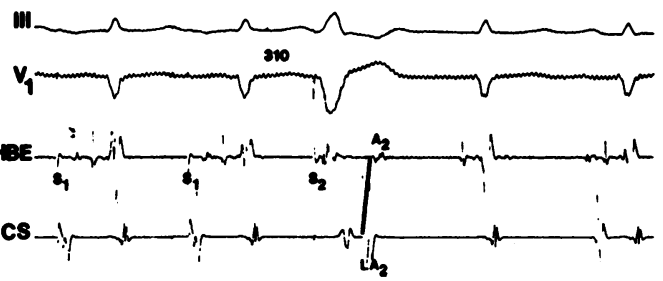

$\mathbf{F}$
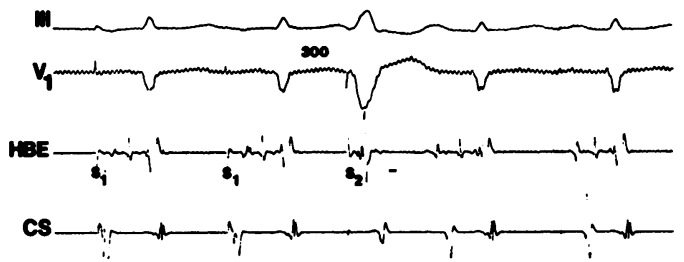

G

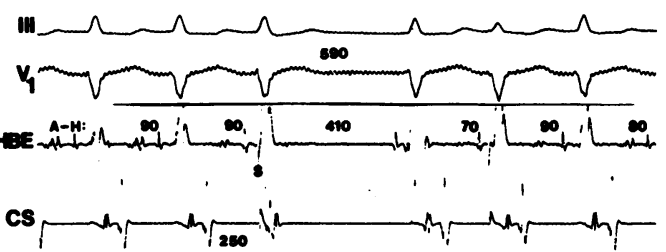

Fig. 6 (case 2) Demonstration of bradycardia-dependent retrograde block in a concealed left-sided atrioventricular accessory pathway (panels A to F). $S_{1}$ represents the last two stimulus artefacts of eight basic drive stimuli delivered to the coronary sinus at a cycle length of $500 \mathrm{~ms} . S_{2}$ is delivered to the right ventricular apex. Numbers in the $V_{1}$ leads are coupling times $\left(V_{1}-V_{2}\right.$ intervals $)$ of the ventricular extrasystoles. $A_{2}$ and $L A_{2}$ are the low right atrial and left atrial deflections conducted from $V_{2}$. The ventricular extrasystoles in each panel are followed by a sinus escape except for panel $\mathrm{E}$ where there is a ventricular echo. The range of coupling times with retrograde conduction over the accessory pathway is from 340 to $310 \mathrm{~ms}$. Panel $\mathrm{G}$ shows that (a) exclusive slow pathway anterograde conduction per se is not responsible for retrograde block in the accessory pathway, and (b) the bradycardia-dependent block is rate-related. See detailed explanation in the text. 
stimuli that have atrioventricular conduction times sufficient to overcome refractoriness in the accessory pathway and in the atrium. The range of coupling times of premature atrial impulses capable of initiating the tachycardia (that is the tachycardia zone) is dependent on several factors including the basic drive rate and the site of stimulation in regard to the site of the accessory pathway. ${ }^{6} 1415$ Once a critical coupling time is reached, however, where the premature atrial impulse initiated paroxysmal supraventricular tachycardia, premature stimuli with shorter coupling times usually also induce the tachycardia up to a point where $A_{2}$ fails to conduct to the ventricles. The only exception is the rare case when very early extra impulses result in pronounced intraatrial delay or in a repetitive atrial response which pre-empts conduction over the concealed accessory pathway.

In the two cases described in this paper, the effects of longitudinal dissociation in the atrioventricular node limited the ability to induce the tachycardia with early premature atrial stimuli. In case 1 , a nonsustained supraventricular tachycardia using an accessory pathway was initiated by premature atrial impulses which developed a critical $\mathrm{A}_{2}-\mathrm{H}_{2}$ interval of $115 \mathrm{~ms}$ or longer (Fig. 1). As the premature atrial coupling intervals shortened, atrioventricular nodal conduction lengthened. Eventually an atrioventricular nodal echo occurred. This atrioventricular nodal echo prevented macro re-entry over the accessory pathway by creating refractoriness in the atrium (Fig. 1B). Since the atrial echo caused by atrioventricular nodal re-entry was too early to be conducted to the ventricles, the re-entry process was limited to one single echo and a tachycardia did not develop (Fig. 1B). Significant intra-atrial delay or repetitive atrial responses were not present with early atrial extra impulses. Therefore, it is reasonable to assume that atrioventricular nodal re-entry alone was responsible for limiting the potential tachycardia zone.

In case 2, a critical A-H interval of $140 \mathrm{~ms}$ was required for the atrial premature stimuli to overcome refractoriness in the retrograde pathway. At atrial coupling intervals of $260 \mathrm{~ms}$ or shorter, however, there was a sudden increment in the atrioventricular nodal conduction times (Fig. 5). At these shorter coupling times with long $\mathrm{A}-\mathrm{H}$ intervals neither atrioventricular nodal re-entrant echoes nor re-entry over the accessory pathway were observed (Fig. 3C). Inspection of the atrioventricular nodal conduction curves of this patient (Fig. 5) disclosed the features ascribed to dual atrioventricular nodal pathways. ${ }^{1}$ Failure of re-entry over the accessory pathway at these long $A_{2}-H_{2}$ intervals could not be reasonably related to concealed anterograde penetration of $\mathrm{A}_{2}$ into the accessory pathway or to atrial refractoriness from the premature atrial stimulus. Inability of the early premature stimuli to initiate paroxysmal supraventricular tachycardia proved to result from a bradycardia-dependent retrograde block in the concealed accessory pathway. The "role" of dual atrioventricular conduction in this case was to delay arrival of impulses from early atrial extrasystoles at the ventricular insertion of the bypass tract and thus prevent them from returning to the atria. The inner limit of the bradycardia-dependent block in the accessory pathway proved to be $350 \mathrm{~ms}$ (Fig. 6C). It is reasonable to suspect that in the absence of a sudden jump in the $\mathrm{A}_{2}-\mathrm{H}_{2}$ intervals with short coupling times of premature stimuli the $V_{1}-V_{2}$ intervals of at least some early atrial stimuli would have remained in the responsive zone of the accessory pathway and thus would have initiated the tachycardia. The sudden jump in the $\mathrm{A}_{2}-\mathrm{H}_{2}$ intervals therefore may have limited the potential tachycardia zone with regard to atrioventricular re-entry.

Bradycardia-dependent accessory pathway block had been previously shown to occur in patients with overt pre-excitation. ${ }^{16}$ To our knowledge our case 2 is the first patient reported where a bradycardia-related retrograde block was proved in a concealed atrioventricular bypass tract. If the range of bradycardia-dependent retrograde block is "far to the right", that is retrograde block in the bypass tract occurs only late in diastole, right ventricular stimulation studies may not be very helpful in disclosing this peculiar disturbance. Because of the proximity of the normal atrioventricular pathway to the right ventricular pacing site, late coupled ventricular extrasystoles may result in atrial activation over the normal pathway even in the presence of a remote left-sided concealed pathway. Therefore, detection of a bradycardia-related block in the accessory pathway with late coupled $V_{2}$ 's may be difficult. In this case, discontinuous atrioventricular nodal conduction curves, as in our patient, may help to show a bradycardia-related retrograde block in a concealed accessory pathway.

Previous studies have shown that in the presence of a concealed atrioventricular bypass tract a sudden increase in the atrioventricular nodal conduction time of early premature atrial stimuli can help to initiate a supraventricular tachycardia. ${ }^{9}$ Moreover, atrioventricular nodal re-entry may be the mechanism of the paroxysmal supraventricular tachycardia in some of these patients. ${ }^{8}$ Our study shows that atrioventricular nodal conduction over a slow pathway and atrioventricular nodal re-entry can also limit the potential tachycardia zone in patients with concealed accessory bypasses. Pharmacological interventions eliminating the above phenomena could paradoxically 
enhance the occurrence of paroxysmal supraventricular tachycardia in these patients.

\section{References}

1 Rosen KM, Denes P, Wu D, Dhingra RC. Electrophysiological diagnosis and manifestation of dual $\mathrm{A}-\mathrm{V}$ nodal pathways. In: Wellens $\mathrm{HJJ}$, Lie $\mathrm{KI}$, Janse $M J$, eds. The conduction system of the heart. Philadelphia: Lea \& Febiger, 1976: 453-66.

2 Cranefield PF. The conduction of the cardiac impulse. Mount Kisco, New York: Futura Publishing Company, 1975: 153-97.

3 Spurrell RAJ, Krikler D, Sowton E. Two or more intra AV nodal pathways in association with either a James or Kent extranodal bypass in 3 patients with paroxysmal supraventricular tachycardia. $B r$ Heart $f$ 1973; 35: 113-22.

4 Zipes DP, DeJoseph RL, Rothbaum DA. Unusual properties of accessory pathways. Circulation 1974; 49: 1200-11.

5 Pritchett ELC, Prystowsky EN, Benditt DG, Gallagher JJ. "Dual atrioventricular nodal pathways" in patients with Wolff-Parkinson-White syndrome. Br Heart $\mathcal{F}$ 1980; 43: 7-13.

6 Farshidi A, Josephson ME, Horowitz LN. Electrophysiologic characteristics of concealed bypass tracts: clinical and electrocardiographic correlates. Am $\mathcal{F}$ Cardiol 1978; 41: 1052-60.

7 Gmeiner $\mathrm{R}, \mathrm{Ng} \mathrm{CK}$, Gstöttner $\mathrm{M}$. Concealed accessory pathway and dual AV conduction: drug-dependent reentrant tachycardia. Clin Cardiol 1979; 2: 291-6.

8 Motté G, Belhassen B, Bodereau P. Syndrome de Wolff-Parkinson-White patent ou inapparent associé à une dualité de conduction nodale. Arch Mal Coeur 1979; 72: $231-40$.
9 Sung RJ, Styperek JL. Electrophysiologic identification of dual atrioventricular nodal pathway conduction in patients with reciprocating tachycardia using anomalous bypass tracts. Circulation 1979; 60: 1464-76.

10 Coumel $P$, Waynberger $M$. Les tachycardies par rythme réciproque au cours du syndrome de Wolff-ParkinsonWhite. Coeur Med Interne 1972; 11: 77-93.

11 Svenson RH, Miller HC, Gallagher JJ, Wallace AG. Electrophysiological evaluation of the Wolff-ParkinsonWhite syndrome. Problems in assessing antegrade and retrograde conduction over the accessory pathway. Circulation 1975; 52: 552-62.

12 Coumel P, Attuel P. Reciprocating tachycardia in overt and latent preexcitation. Influence of functional bundle branch block on the rate of the tachycardia. Eur $\mathcal{F}$ Cardiol 1974; 1: 423-36.

13 Ward DE, Camm AJ, Spurrell RAJ. Patterns of atrial activation during right ventricular pacing in patients with concealed left-sided Kent pathways. Br Heart $\mathcal{f} 1979 ; 42$ : 192-200.

14 Denes P, Wu D, Amat-y-Leon F, et al. Determinants of atrioventricular reentrant paroxysmal tachycardia in patients with Wolff-Parkinson-White syndrome. Circulation 1978; 58: 415-25.

15 Pritchett ELC, Gallagher JJ, Scheinman M, Smith WM. Determinants of antegrade echo zone in the WolffParkinson-White syndrome. Circulation 1978; 57: 671-7.

16 Przybylski J, Chiale PA, Quinteiro RA, Elizari MV, Rosenbaum MB. The occurrence of phase-4 block in the anomalous bundle of patients with Wolff-ParkinsonWhite syndrome. Eur f Cardiol 1975; 3: 267-80.

Requests for reprints to Dr Laszlo Littmann, 3rd Department of Medicine, Semmelweis University Medical School, Eotvos utca 12, Budapest 1121, Hungary. 